\title{
Spiral windows for escaping stars
}

\author{
J. F. Navarro ${ }^{1}$ and J. Henrard ${ }^{2}$ \\ 1 Depto. de Matemática A., University of Alicante, PO Box 99, 03080, Alicante, Spain \\ 2 Dépt. de Mathématique, University of Namur, 8 rempart de la Vierge, 5000, Namur, Belgium
}

Received 27 November 2000 / Accepted 23 January 2001

\begin{abstract}
The "escape windows" through which stars may escape from a galaxy are investigated by means of Poincaré sections of a simplified axi-symmetric model of the potential. The spiral shape of the windows are analyzed in details by constructing a normal form in the vicinity of an unstable periodic orbit.
\end{abstract}

Key words. celestial mechanics - galaxies: clusters: general - galaxies: general

\section{Introduction}

The aim of the paper is to investigate the shape of the windows through which test particles; may escape the dynamics of which is controlled by the Hamiltonian

$\mathcal{H}=\frac{1}{2}\left(\dot{x}^{2}+\dot{y}^{2}\right)+\frac{1}{2}\left(x^{2}+y^{2}\right)-\mu x^{2} y^{2}$.

The potential $W(x, y)=\left(x^{2}+y^{2}\right) / 2-\mu x^{2} y^{2}$ may represent the central part of deformed axisymmetric galaxies and has been extensively studied (see Barbanis 1990; Contopoulos 1990; Contopoulos \& Kaufmann 1992; Siopsis et al. 1997 and references therein), especially with respect to orbits escaping from the potential well. It is actually a member of the Verhulst family of galactic potentials (see Verhulst 1979 for a review). Another member led to the well known Hénon and Heiles paper on the third integral of motion (Hénon \& Heiles 1964). Actually, the parameter $\mu$ could be scaled away by changing both the unit of length and the unit of time. However, we have kept it for the sake of comparison with earlier investigations.

There is a critical value of the energy $(h=1 / 4 \mu)$ such that, for larger values of $h$, the potential well opens up to infinity and test particles may escape. Due to the symmetries of the potential, the well opens up at four places along the diagonals of the configuration space $(x= \pm y)$ (see Fig. 1). For each larger value of $h$, there is an unstable periodic orbit across the opening, bouncing back and forth between the two "walls" of the pass.

It has long been recognized (see for instance Contopoulos 1990) that these unstable periodic orbits are, in some sense, the guardians of the pass: orbits going through the pass are sheperded by the stable and unstable manifolds of the periodic orbit. This can be explained

Send offprint requests to: J. Henrard,

e-mail: jacques.henrard@fundp.ac. be

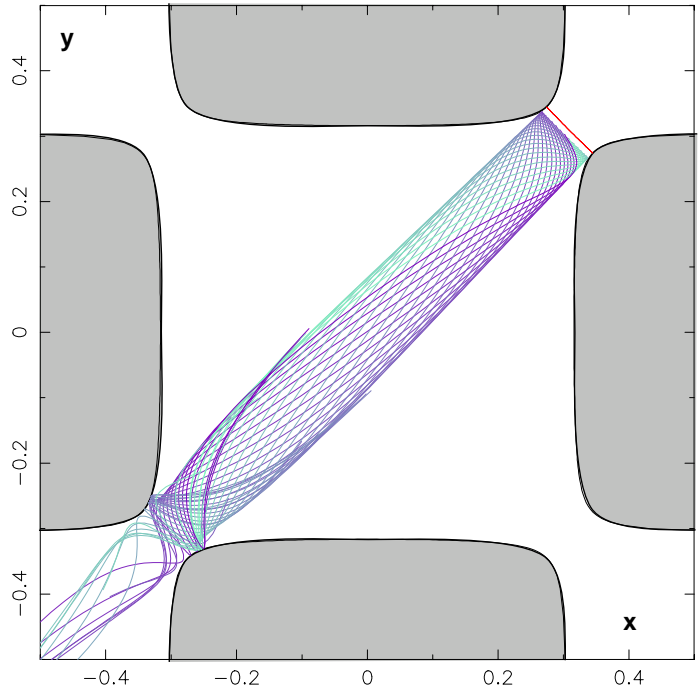

Fig. 1. The stable manifold to the periodic orbit "guarding" the upper-right opening in the potential well for $\mu=5.26$ and $h=0.05$. The guardian orbit is the almost straight line barring the opening. The stable manifold is computed backward in time starting from initial conditions computed from a sixth order approximation. One can see that some of the orbits come from the lower-left opening, but others come from the potential well after bouncing on the zero velocity curve

in the following way: a continuous variation of initial conditions may lead from an orbit which "bounces back" in the pass and returns inside the well to an orbit which passes through, a very non-continuous behavior. The only way this can happen is that somewhere between these two orbits there is a third one which stays in the pass for an infinite amount of time. Indeed, the general solution $\boldsymbol{X}(\boldsymbol{x}(0), t)$ is a continuous function of the initial condition $\boldsymbol{x}(0)$ for any finite time $t$. 
Hence, in order to investigate the size, shape and properties of the regions of phase space leading to escape, it is necessary to understand the geometry of the stable manifolds to the "guardian" periodic orbits. We will do so by investigating the intersections of the stable and unstable manifolds with a surface of section. This has been the strategy of Contopoulos et al. (Contopoulos 1990; Contopoulos \& Kaufmann 1992). They found numerically that some of the intersections take the form of narrow bands spiraling infinitely around a "limiting curve". They related this behavior to the presence of heteroclinic orbits.

We revisit this method, clarify the relation between the infinite spiraling and heteroclinic orbits and, furthermore describe a new feature: there are first order spirals, as described by them, but there are also second order spirals which are formed of an infinite number of spirals, embedded in each other like Russian dolls, and then third order spirals, formed by an infinite number of second order spirals, and so on.

Unlike Contopoulos et al., we take as surface of section the plane $x=0$ and not the plane $x=y$. Qualitatively both sections are equivalent, but the one we chose makes it easier to distinguish the various returns to the section and makes this count independent of the initial conditions chosen on the stable or unstable manifold. Another difference between their investigation and ours is that we use a sixth order expansion of the stable and unstable manifolds in order to obtain precise initial conditions far enough from the unstable "guardian orbit" to start a safe computation of these manifolds. They used a first order approximation, but then had to use a quadruple precision numerical integration to cover with accuracy the part of the orbits close to the guardian orbit.

The spiral structures shown here are generic and closely related to the "chaotic" scattering phenomenon. It is well known (see for instance Moser 1973) that homoclinic or heteroclinic orbits are associated with hyperbolic sets leading to chaos and fractal dimensions of the boundaries between classes of motion. Chaotic scattering problems show this behavior and have been extensively studied numerically and analytically (see for instance Noid et al. 1985; Petit \& Hénon 1986; Bleher et al. 1990; Ott 1993). Although it is rarely explicitly pointed out, the computed Poincaré sections, in most instances, show spiral structures. Our analysis describes in detail these structures and confirms that they are the inescapable signature of the presence of homoclinic or heteroclinic orbits.

\section{Symmetries of the problem}

The phase space of Hamiltonian (1) is symmetric with respect to each of the two coordinates $(x, y)$ (and their velocities) and with respect to time (and velocities) reversal, i.e. if $(x(t), y(t), \dot{x}(t), \dot{y}(t))$ is an orbit, then the following are also orbits

$$
\begin{array}{lrrrr}
\text { (a) } & x(t), & y(t), & \dot{x}(t) & , \dot{y}(t), \\
\text { (b) } & -x(t), & y(t), & -\dot{x}(t) & , \dot{y}(t), \\
\text { (c) } & x(t), & -y(t), & \dot{x}(t), & -\dot{y}(t), \\
\text { (d) } & -x(t), & -y(t), & -\dot{x}(t), & -\dot{y}(t), \\
& & & \\
\text { (e) } & x(-t), & y(-t), & -\dot{x}(-t), & -\dot{y}(-t), \\
\text { (f) } & -x(-t), & y(-t), & \dot{x}(-t), & -\dot{y}(-t), \\
\text { (g) } & x(-t), & -y(-t), & -\dot{x}(-t), & \dot{y}(-t), \\
\text { (h) } & -x(-t), & -y(-t), & \dot{x}(-t), & \dot{y}(-t) .
\end{array}
$$

Hence it is enough to compute the sections of the stable manifold to one periodic orbit (let us take as (a) the stable manifold to the periodic orbit in the upper-right corner see Fig. 1) in order to know by symmetry the sections of the stable and unstable manifolds of the four guardian periodic orbits. This is shown in Fig. 2.



Fig. 2. The first intersections of the two surfaces of section $(x=0 ; \dot{x}>0)$ and $(x=0 ; \dot{x}<0)$ with the symmetrics (enumerated in Eq. (2)) of the (internal part of the) stable manifold. The areas with vertical hatching correspond to orbits coming from "outside" and which, as a consequence, do not have any antecedents on the surfaces of section. Areas with horizontal hatching correspond to orbits leaving the potential well. Of course areas with both hatching correspond to orbits "just passing through" the potential well

The symmetries of the problem also lead to a very degenerate family of particular solutions along the main diagonals. Let us consider the one degree of freedom differential system

$\ddot{z}=-z+2 \mu z^{3}$.

To each solution of (3), a solution corresponds along one of the diagonals of the system (1) with $x=y=z$ or $x=-y=z$. The one degree of freedom problem (3) is easy to analyze. It possesses two unstable equilibria for $z= \pm 1 / 2 \mu$. For each value of the energy below the value $E=1 / 8 \mu$ reached at the equilibria, there is a periodic orbit bouncing back and forth between the walls of the 


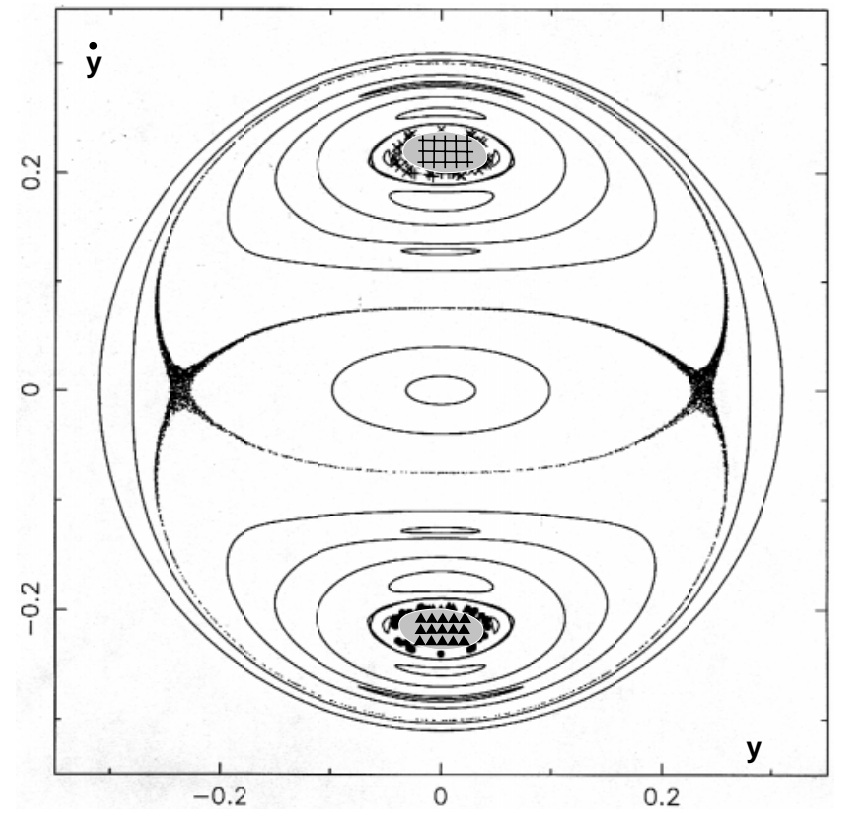

Fig. 3. Surface of section for $h=0.048$, just above the critical value. The "main escape windows" (initial conditions of orbits immediately leaving the potential well) are surrounded by orbits leaving the potential well after several crossings of the surface of section. The first quadrant main window (upper part of the figure) is surrounded by escapers to the first $(+)$ and third $(x)$ quadrant, while the fourth quadrant main window is surrounded by escapers to the second $(\bullet)$ and fourth $(\triangle)$ quadrant. The two main windows and their immediate surroundings are well separated by regular invariant tori

potential well centered at $z=0$. For $E=1 / 8 \mu$ we find an heteroclinic orbit

$z=\frac{1}{\sqrt{2 \mu}} \frac{\exp (\sqrt{2} t)-1}{\exp (\sqrt{2} t)+1}$

joining the two unstable equilibria. Barbanis (1990) has shown how the family of periodic orbits changes stability an infinite number of times while approaching the critical value of $E$. If $E_{k}$ are the values of the energy at which the family changes stability while approaching the heteroclinic orbit (for $k \longrightarrow \infty$ ), then the fraction $\left(E_{k}-E_{k-1}\right) /\left(E_{k+1}-E_{k}\right)$ tends to $e^{\pi}$.

This degenerate situation will lead to a non-generic opening of the escape windows of the two degrees of freedom system when the energy level goes above the critical value (see Sect. 8)

\section{Surfaces of section and the opening of the escape windows}

Let us take the hyperplane $x=0$ as the surface of a section. The intersection of this plane with the four dimensional surface $\mathcal{H}(\dot{x}, \dot{y}, x, y)=h$ is the sphere $2 h=$ $\dot{x}^{2}+\dot{y}^{2}+y^{2}$. The great circle $2 h=\dot{y}^{2}+y^{2}$ corresponds to the periodic orbit bouncing back and forth between the wall of the potential well on the axis $x=0$, while the other special periodic orbit, bouncing back and forth be-

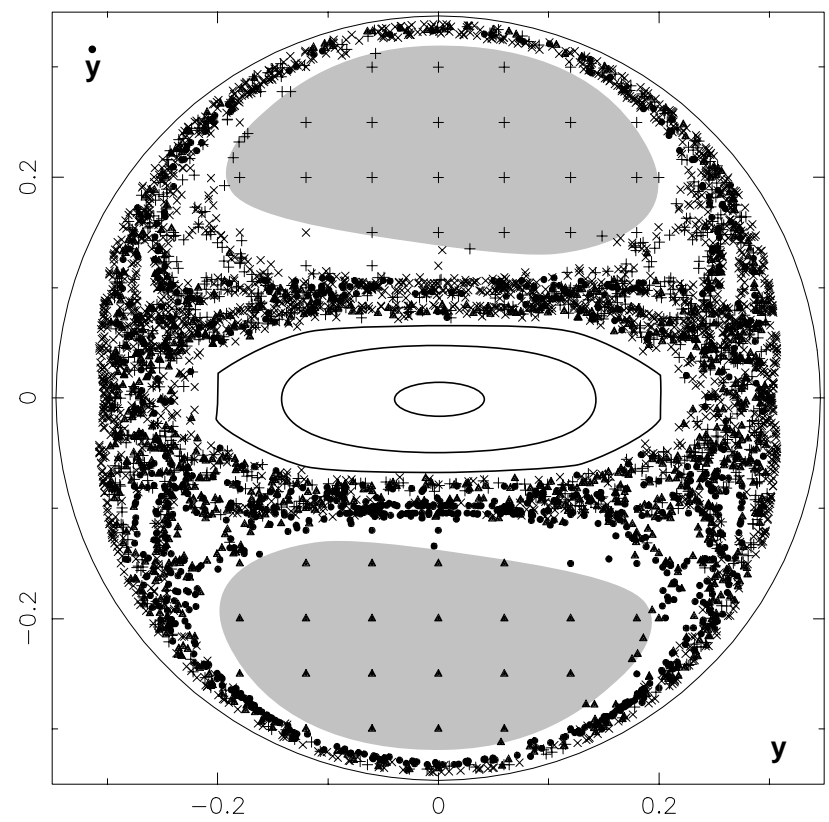

Fig. 4. Surface of section for $h=0.06$. The surroundings of the two main windows are now connected

tween the wall of the potential well on the axis $y=0$ is represented by the two points $(y=0, \dot{y}=0, \dot{x}= \pm \sqrt{2 h})$.

Figure 3 shows the projection on the plane $(y, \dot{y})$ of the hemisphere $(\dot{x}>0)$ of the surface of section for $h=0.048$ (a little above the critical value $h=1 / 4 \mu \approx 0.4753$ ). The other hemisphere can be deduced from it by symmetry. Special signs $(+)$ (respectively $\bullet,(\times), \triangle$ ) correspond to initial points leading to an escape by the first (respectively second, third, fourth) quadrant. We see that small windows of escape open up around the locations of the particular heteroclinic orbits mentioned above. In the upper half plane (resp. the lower half plane), the "main window" consists in orbits leaving immediately through the first (resp. the fourth) quadrant. We see also that even for values of the energy just above the critical value these main windows are surrounded by orbits leaving the potential well after several crossings of the surface of section either through the same quadrant or through the one symmetric with respect to the origin.

Let us emphasize here that, contrary to what is implied on page 61 and 62 of (Siopsis et al. 1997), the escape windows open up immediately after the critical value of $h$ is reached and do not stay closed until a higher empirical critical value is reached.

For values of $h$ small enough, the rest of the surface of section seems to be filled by regular motion, except for the vicinity of the unstable periodic orbit at $(y \approx \pm 0.23, \dot{y}=0)$. The regular invariant tori isolate from each other the two escape windows shown in Fig. 3. For higher values of the energy, the escape windows communicate as shown in Fig. 4, and each "main window" is surrounded by orbits escaping through all the four quadrants. Figure 5 magnifies a small region surrounding the first quadrant window $(-0.02 \leq y \leq 0 ; \quad 0.094 \leq \dot{y} \leq 0.098)$ and shows how the four types of escape are mixed. 


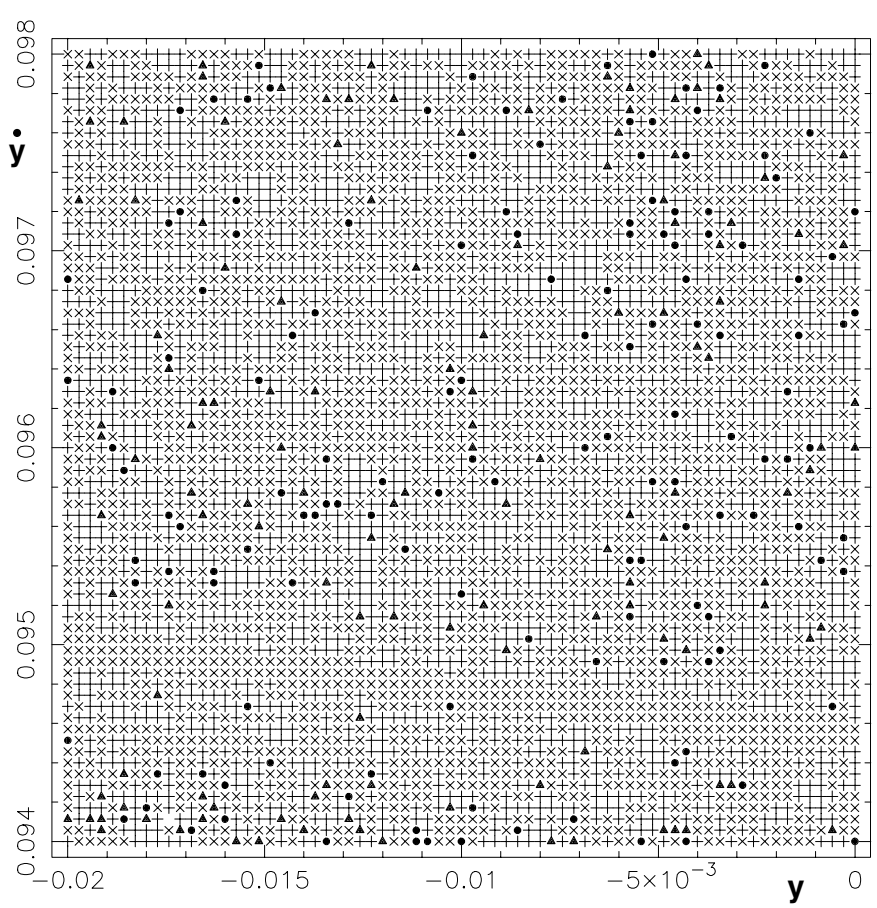

Fig. 5. Detail of the region surrounding the main escape window to the upper-right corner for $h=0.06$. Initial conditions leading to an escape through the upper-right (resp. lower-left, lower-right, upper-right) corner are marked by a cross (resp. a $(\times)$, a $(\bullet)$, a dark triangle)

In the following section, we will investigate in more detail the "secondary windows" (sets of initial conditions of orbits which escape after several crossings of the surface of section) which surround in a very intricate way the main windows. In order to simplify the analysis, we choose a value of the energy, $h=0.05$, for which the surroundings of the main windows are not yet connected.

\section{Computation of the stable and unstable manifolds}

As mentioned in the introduction, the stable manifolds to the "guardian" unstable periodic orbits form the boundaries of the escape windows we wish to analyze.

For the computation of the initial part of these stable manifolds (and of the unstable manifolds as well), we shall follow the scheme proposed by Deprit \& Henrard (1969). The stable manifold to a periodic orbit $\left(x^{*}(t), y^{*}(t)\right)$ of period $T$ is represented by series

$$
\begin{aligned}
& x(t, \epsilon)=x^{*}(t)+\epsilon u(t) ; u(t)=\sum_{j \geq 1} \epsilon^{j-1} \mathrm{e}^{-\mathrm{jat}} x_{j}(t) \\
& y(t, \epsilon)=y^{*}(t)+\epsilon v(t) ; v(t)=\sum_{j \geq 1} \epsilon^{j-1} \mathrm{e}^{-\mathrm{jat}} y_{j}(t)
\end{aligned}
$$

where the coefficients $x_{j}(t)$ and $y_{j}(t)$ are periodic with period $T$ and $a$ is the (positive) characteristic exponent of the periodic orbit. The unstable manifold is represented by similar series with the exponent $-a$ replaced by $+a$. When $\epsilon$ is equal to zero, we recover the periodic orbit.
Substituting the series (5) in the differential equations derived from the Hamiltonian (1), we obtain

$$
\begin{aligned}
& \ddot{u}=W_{x x}^{*} u+W_{x y}^{*} v+\sum_{i \geq 1} \epsilon^{i-1} \sum_{j \geq 0}^{i} c_{i}^{j}\left(\frac{\partial^{i} W}{\partial x^{j} \partial y^{i-j}}\right)^{*} u^{j} v^{i-j} \\
& \ddot{v}=W_{y x}^{*} u+W_{y y}^{*} v+\sum_{i \geq 2} \epsilon^{i-1} \sum_{j \geq 0}^{i} c_{i}^{j}\left(\frac{\partial^{i} W}{\partial x^{j} \partial y^{i-j}}\right)^{*} u^{j} v^{i-j}
\end{aligned}
$$

where we have used the notation $f^{*}$ for the function $f$ evaluated along the periodic solution $\left[f^{*}(t)=f\left(x^{*}(t), y^{*}(t)\right)\right]$. Expanding $u(t)$ and $v(t)$ in powers of $\epsilon$, the Eq. (6) lead to a recursive set of linear equations for the coefficients $x_{k}(t)$ and $y_{k}(t)$

$$
\begin{aligned}
k^{2} a^{2} x_{k}+2 k a \dot{x}_{k}+\ddot{x}_{k} & =W_{x x}^{*} x_{k}+W_{x y}^{*} y_{k}+\Phi_{k}(t) \\
k^{2} a^{2} y_{k}+2 k a \dot{y}_{k}+\ddot{y}_{k} & =W_{x y}^{*} x_{k}+W_{y y}^{*} y_{k}+\Psi_{k}(t)
\end{aligned}
$$

where the periodic functions $\Phi_{k}(t)$ and $\Psi_{k}(t)$ are the contributions of the summations over $j$ in (6) multiplied by $e^{\text {jat }}$. These contributions can readily be computed at order $k$ and are periodic if the $x_{\ell}(t)$ and $y_{\ell}(t)$ (for $\ell<k$ ) are known and periodic.

For $k=1$ the functions $\Phi_{k}(t)$ and $\Psi_{k}(t)$ vanishes and the system (6) is singular. A non-trivial solution is given by the solution of the linear system of variational equations corresponding to the eigenvalue $a$ of the principal matrix. In order to compute it we integrate numerically the two isoenergetic solutions of the variational equations, the initial conditions of which are perpendicular to the initial velocity of the periodic orbit (see Deprit \& Henrard 1969). In this way we avoid the eigenspace corresponding to the double zero-eigenvalue. The initial conditions are $\left[u(0)=v(0)=0, \dot{u}(0)=-\dot{y}^{*}(0), \dot{v}(0)=\dot{x}^{*}(0)\right]$, and $\left[u(0)=-\dot{y}^{*}(0), v(0)=\dot{x}^{*}(0), \dot{u}(0)=\sigma \dot{x}^{*}(0)\right.$, $\left.\dot{v}(0)=\sigma \dot{y}^{*}(0)\right]$ with $\sigma=\left(\dot{x}^{*}(0) W_{y}^{*}(0)-\dot{y}^{*}(0) W_{x}^{*}(0)\right) / V^{3}$ where $V$ is the initial velocity on the periodic orbit. We select as solution the linear combination of these two variations which, after a period $T$, reproduces its initial condition multiplied by the factor $\exp (a T)$.

The periodic orbit and this solution of the variational equations are expanded in Fourier series and thereby the recursive Eq. (6) can be written as a recursive set of linear systems for the Fourier coefficients of the periodic functions $x_{j}(t)$ and $y_{j}(t)$.

In order to illustrate the convergence of these series, we show, in Table 1, the coefficients of the Cosine part of the Fourier series for the $x_{j}(t)$ up to order 6 . The Sine part has a similar behavior. The periodic orbit analyzed corresponds to $\mu=5.26$ and to the energy $h=0.05$. Its period is $T=4.445768263501$ and its characteristic exponent is $a=1.419732239168$.

The Fourier part of the expansion converges quickly, while the power series in $\epsilon \exp (a t)$ behave roughly as $0.3 \sum[\epsilon \exp (a t) / 0.7]^{n}$. If we aim at an accuracy of $10^{-12}$ for the initial conditions, we can evaluate the gain obtained by truncating at orders higher than one in the following way. The interest of truncating at higher order is to 
Table 1. Fourier coefficients of the even part of the functions $x_{j}(t)$ for $j=0,2,4,6$ showing the convergence of the Fourier series and of the power series in $\epsilon$

\begin{tabular}{rcccc}
\hline$J$ & \multicolumn{1}{c}{$X_{0}^{\mathrm{c}}$} & \multicolumn{1}{c}{$X_{2}^{\mathrm{c}}$} & \multicolumn{1}{c}{$X_{4}^{\mathrm{c}}$} & \multicolumn{1}{c}{$X_{6}^{\mathrm{c}}$} \\
\hline 0 & 0.309 & 0.807 & \multicolumn{1}{c}{1.052} & \multicolumn{1}{c}{1.372} \\
1 & $0.15110^{-07}$ & $0.90410^{-02}$ & $-0.12710^{-01}$ & \multicolumn{1}{c}{$-0.015310^{-01}$} \\
2 & $-0.20110^{-03}$ & $0.33210^{-03}$ & $-0.10810^{-03}$ & $0.21710^{-03}$ \\
3 & $0.73710^{-11}$ & $-017710^{-04}$ & $0.11710^{-04}$ & $-0.19110^{-05}$ \\
4 & $-0.58110^{-08}$ & $0.65910^{-07}$ & $-0.78210^{-07}$ & $0.72010^{-07}$ \\
5 & $0.45010^{-14}$ & $-0.75310^{-08}$ & $0.23010^{-08}$ & $-0.39910^{-08}$ \\
6 & $0.77010^{-12}$ & $0.16610^{-10}$ & $-0.47410^{-10}$ & $0.41910^{-10}$ \\
7 & $0.10010^{-14}$ & $-0.23110^{-11}$ & $-0.44310^{-11}$ & $0.54710^{-11}$ \\
8 & $0.90010^{-15}$ & $-0.77110^{-13}$ & $-0.24810^{-12}$ & $-0.23910^{-12}$ \\
9 & $0.40010^{-15}$ & $-0.13710^{-13}$ & $-0.69410^{-13}$ & $-0.74510^{-13}$ \\
10 & $0.40010^{-15}$ & $0.94010^{-14}$ & $0.20610^{-13}$ & $0.45510^{-13}$ \\
11 & $0.40010^{-15}$ & $0.83010^{-14}$ & $0.25410^{-13}$ & $0.49110^{-13}$ \\
12 & $0.30010^{-15}$ & $0.7510^{-14}$ & $0.22110^{-13}$ & $0.36110^{-13}$ \\
13 & $0.20010^{-15}$ & $0.10110^{-13}$ & $0.31110^{-13}$ & $0.49110^{-13}$ \\
14 & $0.10010^{-15}$ & $0.11810^{-13}$ & $0.41010^{-13}$ & $0.72610^{-13}$ \\
15 & $<0.510^{-16}$ & $0.98010^{-14}$ & $0.36710^{-13}$ & $0.70010^{-13}$ \\
16 & $<0.510^{-16}$ & $0.89010^{-14}$ & $0.34810^{-13}$ & $0.69010^{-13}$ \\
\hline & & & &
\end{tabular}

cover analytically a larger portion of the manifolds. This can be measured in unit of time. Table 2 shows the values of $t_{n}^{*}$, the time necessary to go from the initial conditions corresponding to $\epsilon_{1}=8 \cdot 10^{-7}$ (giving an accuracy of $10^{-12}$ to the first order approximation) to the initial conditions corresponding to $\epsilon_{n}$ (giving an accuracy of $10^{-12}$ to the $n$-order approximation).

Table 2. The "gain in time" when using an approximation of higher order than the first. The length of time $t_{n}^{*}$ is covered analytically and does not have to be integrated numerically

\begin{tabular}{c|ccccccc}
\hline$n$ & 2 & 4 & 6 & 8 & 10 & 12 & $\infty$ \\
$\epsilon_{n} / \epsilon_{1}$ & $210^{2}$ & $610^{3}$ & $310^{4}$ & $710^{4}$ & $110^{5}$ & $210^{5}$ & $910^{5}$ \\
$t_{n}^{*}$ & 3.7 & 6.2 & 7.2 & 7.8 & 8.2 & 8.5 & 9.6 \\
\hline
\end{tabular}

As expected, we see a very strong law of diminishing return. Furthermore, the complexity of the computation of the series is proportional to the third power of the order and the accumulation of round-off errors may become worrisome for very high orders. Round-off errors are indeed already apparent in the tail of the Fourier series in Table 1 ; but at this stage they are harmless as the Fourier series $X_{n}$ is multiplied by $\epsilon^{n}$. In view of these observations, we chose to truncate at order 6 and to give to $\epsilon \exp (a t)$ the value 0.01 .

\section{The boundaries of the escape windows}

We integrate numerically and backward (using Lie series of order 20 - see Deprit \& Price 1969) some 10000 or-
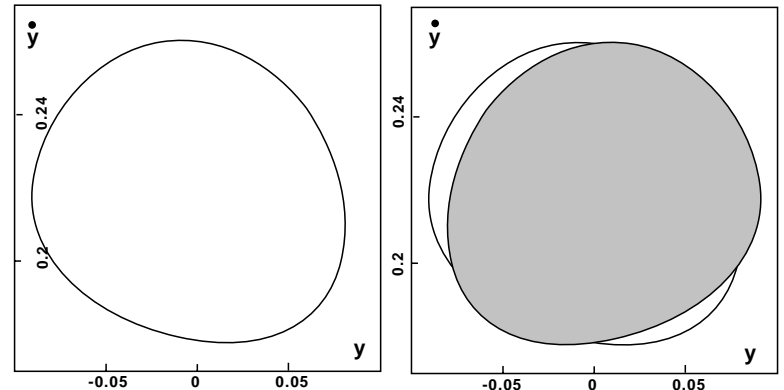

Fig. 6. First section (by $x=0 ; \dot{x}>0$ ) of the stable manifold (a) to the first quadrant guardian periodic orbit (on the left panel). On the right panel we have also reproduced (in grey), the section of the unstable manifold $(h)$ to the third quadrant periodic orbit. The area inside both sections corresponds to orbit coming from infinity from lower left, passing through the potential well once, and disappearing to infinity to the upper right. The two remaining crescents will have antecedents on the surface of section (see Fig. 7)
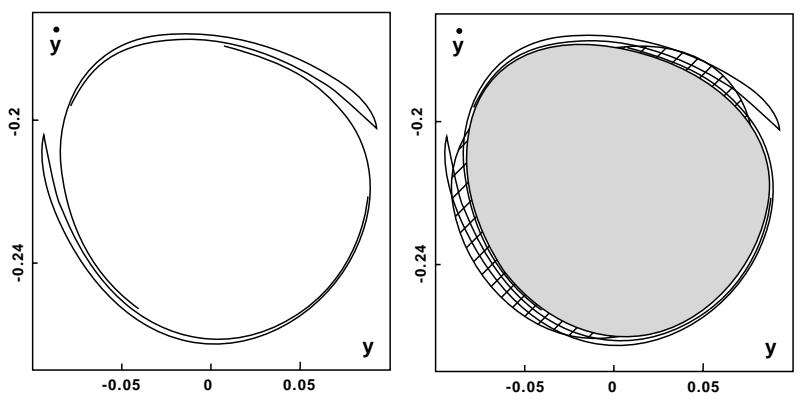

Fig. 7. Second section (by $x=0 ; \dot{x}<0$ ) of the stable manifold to the upper-right guardian periodic orbit (at left). The section is composed of two tongues, images of the two crescent of the right panel of Fig. 6, winding around the stable manifold $(d)$ (shown in grey on the right panel). The explanation for this infinite winding will be given in Sect. 6. Also shown (surrounding the dashed area) is the section of the unstable manifold $(e)$ to the upper-right guardian

bits belonging to the stable manifold to the first quadrant guardian, until they cross the hyperplane $x=0$ (with $\dot{x}>0$ ). The initial conditions were computed from the series (5) by keeping the value of $\epsilon \exp (a t)$ fixed at 0.01 and choosing 10000 equidistant values of the initial time $t$. The result is shown in Fig. 6. By symmetry we can also plot the trace of the unstable manifold to the third quadrant guardian (called $h$ in Fig. 2). We observe that the two "rings" intersect. Orbits starting inside both rings are coming from the lower-left infinity and are going to the upper right infinity. They just pass through the center of the galaxy. On the other hand, orbits starting from one of the two crescents shown in the right panel of Fig. 6 originate inside the galaxy (and of course escape in the future by the upper-right window), and we can consider their previous intersections with the surface of section.

The second intersection, shown in Fig. 7 (left panel), is composed of two tongues which spiral around the stable manifold to the third quadrant guardian orbit (called $d$ in Fig. 2). As shown in Sect. 6, the spirals are infinite, but 


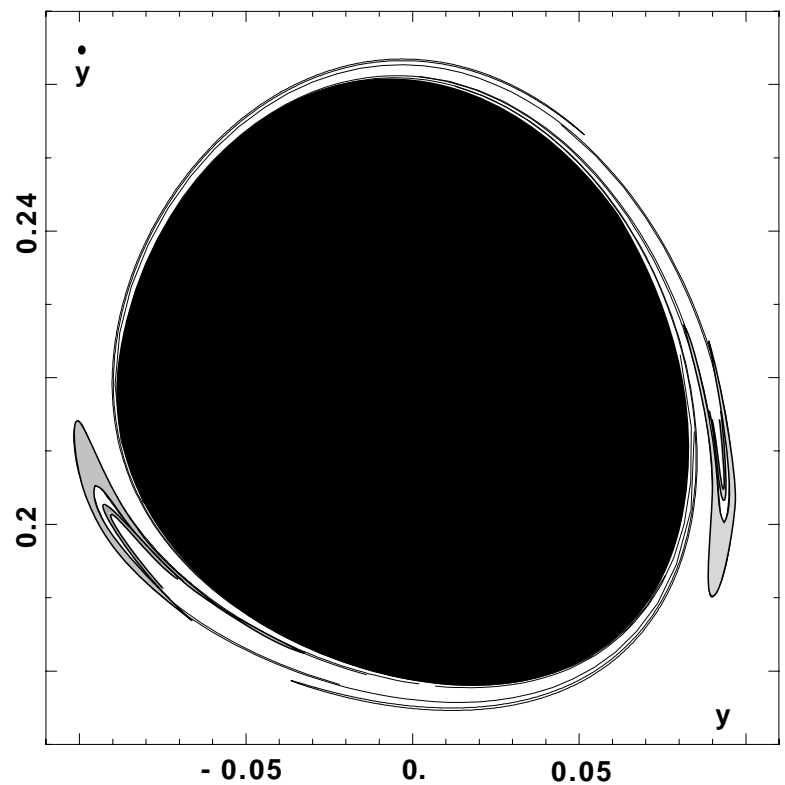

Fig. 8. Third section (by $x=0 ; \dot{x}>0$ ) of the stable manifold to the first quadrant periodic orbit. The section is composed of two "simple" tongues (the very narrow ones) plus two complex ones. The complex ones are "russian dolls", composed of an infinity of "subtongues" embedded inside each other. All these tongues wind around the stable manifold $(a)$ to the first quadrant guardian, shown in black and reproduced from the left panel of Fig. 6

of course we have computed (and shown in Fig. 7) only a small part of them. We show also in the right panel the ring created by the unstable manifold to the first quadrant orbit (called $e$ in Fig. 2). Orbits starting inside this ring, and inside one of the two tongues, come from infinity by the upper-right window and leave the galaxy by the same window after two crossings of the axis $x=0$. Orbits inside the tongues but outside the dashed area of Fig. 7 (right panel) have a previous intersection with the surface of section.

Following these latter orbits backward, we compute the third section (see Fig. 8). It is composed of two "simple" tongues (the very narrow ones) plus two complex ones. The complex ones are "russian dolls", composed of "subtongues" embedded inside each other. Only a few of these subtongues have been computed. As explained in Sect. 6, there are an infinity of those subtongues. All these tongues are infinitely winding around the stable manifold $(a)$ to the first quadrant guardian (already shown in Fig. 6). Orbits starting inside this latter manifold (in the area shown in black) are those which escape directly by the first quadrant window. The fate of the orbits starting inside the complex tongues alternates according to the parity of the number of subtongues in which they are embedded. Those starting from the area shown in gray in Fig. 8 are mapped on the pieces of the tongues of Sect. 2 which are not inside the dashed area (Fig. 7 - right panel) and thus escape by the upper-right window after two crossing of the surface of section. The fate of the other is not decided at this stage. We did not reproduce in Fig. 8 the trace of the unstable manifold to the third quadrant periodic orbit (shown in grey in the right panel of Fig. 6) because the figure is already difficult to understand; but, of course, it is present and intersects the tongues. Orbits inside it and inside the tongues come from infinity by the lower-left window; the others have antecedents on the surface of section and we could compute them backward to a fourth intersection with the surface of section.

While we have computed and drawn the third intersection in spite of its complexity, we have renounced to do the same with the fourth one. Indeed, each tongue and subtongue of Fig. 8 is cut an infinite number of times by the area shown in grey in the right panel of Fig. 6. Only the pieces which do not fall in this area can be followed back. Hence, the fourth intersection is composed of an infinity of subtongues embedded in each other and winding infinitely; each of the subtongues being itself composed of an infinity of "sub-subtongues" embedded in each other. In 1899, Poincaré did not dare draw the "réseau à mailles infiniment serrées" of the crossing of a stable and an unstable manifold. In 1999, we are facing a similar problem.

\section{Normalization in the vicinity of the unstable periodic orbits}

We show in Appendix II that in the vicinity of an unstable periodic orbit of a Hamiltonian system $\mathcal{H}\left(x_{1}, x_{2}, x_{3}, x_{4}\right)$, the Hamiltonian $\mathcal{H}$ can be normalized into

$\mathcal{P}\left(u_{1}, u_{2}, u_{3}, u_{4}\right)=a U-\mathcal{R}\left(U, u_{2}, u_{4}\right)$,

with

$\mathcal{R}=\frac{1}{2} u_{2}^{2}+\sum_{i \geq 1} R_{i}\left(U, u_{4}\right)$

where $a$ is the positive multiplier of the unstable periodic orbit and where the product $U=u_{1} u_{3}$ and the function $\mathcal{R}$ are both constants of motion. The stable manifold is caracterized by $\left(u_{1}=u_{2}=u_{4}=0\right)$ and the unstable manifold by $\left(u_{3}=u_{2}=u_{4}=0\right)$. The function $\mathcal{R}$ can be considered as the Hamiltonian of a one degree of freedom problem depending on the parameter $U$. Notwithstanding the fact that the algorithms proposed do not generate small denominators, we do not know whether the generating formal power series $\mathcal{W}=\sum \mathcal{W}_{k}$ and $\mathcal{V}=\sum \mathcal{V}_{k}$ converge. But, as we use the result of this normalization only for developing good approximations of the motion in the vicinity of the unstable periodic orbit, a truncated normalization will suffice.

We are interested mainly by orbits, starting with $u_{4}=0$, for very small $U$, and for $\mathcal{P}=0$ (same level of energy as the periodic orbit). Let us define a vicinity of the periodic orbit

$\mathcal{C}=\left\{0 \leq U \leq 2 \epsilon ; 0 \leq u_{2} \leq \sqrt{5 a \epsilon} ; 0 \leq u_{4} \leq \epsilon^{1 / 3}\right\}$

As long as an orbit stays in $\mathcal{C}$, the velocities $\dot{u}_{2}$ and $\dot{u}_{4}$ are respectively of the order of $U$ and $\sqrt{U}$. Hence if the 

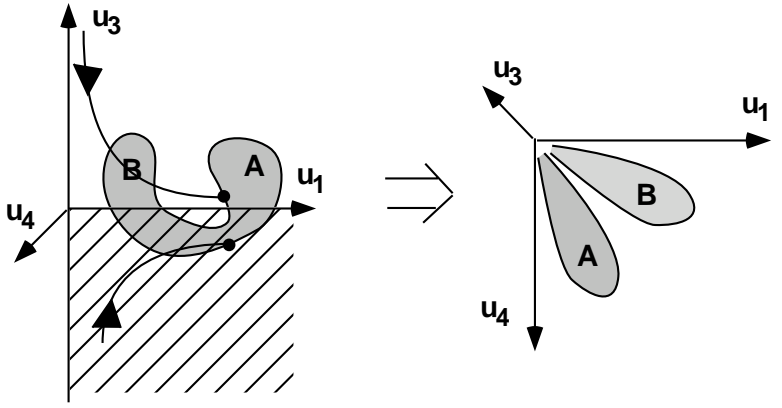

Fig. 9. Passage close to the unstable periodic orbit in the third quadrant, between the first and the second intersections with the surface of section (see Figs. 6 and 7). To the left we show the section by the plane $u_{4}=0$ just before the close approach to the periodic orbit (located at the origin); to the right, the section by the plane $u_{3}=u_{3}^{*}$, just after the close approach

initial conditions are $u_{1}=\delta, u_{2} \leq \sqrt{3 a \epsilon}, u_{3}=\epsilon$, and $u_{4}(0)=0$, the orbit remains in $\mathcal{C}$ at least for a time span as large as $(2 / a) \log \delta / \epsilon$, which is enough to go from the initial condition to the plane $u_{3}=u_{3}^{*} \leq \delta$ (see the right panel of Fig. 9).

\section{Interpretation of the numerical results}

With the help of the analysis conducted in the previous section, we will now show how the numerical results of Sect. 5 can be explained.

Let us consider the intersection of the stable manifold labeled $(a)$ in Fig. 2 with a surface of section, close to the lower-left periodic orbit and defined in the following way. Let

$\boldsymbol{x}=\boldsymbol{x}^{*}\left(t_{0}\right)+\mathcal{T}\left(t_{0}, u_{1}, u_{2}, u_{3}, u_{4}\right)$,

be the normalizing canonical transformation defined in the previous section for the third quadrant periodic orbit and a given initial time $t_{0}$. We define the surface of section as the plane $u_{4}=0$. The flow, in the vicinity of the periodic orbit, is transverse to it because $\dot{x}_{0}^{*}$ is a eigenvector corresponding to the unit eigenvalue of $\Psi(T)$ (see Appendix I) and is thus sent to the axis $u_{4}$. The plane $u_{4}=0$ is thus perpendicular to the flow on the unstable manifold and transverse to it in the vicinity. We restrict ourselves to orbits with the same energy and consider that, for given values of $\left(u_{1}, u_{3}, u_{4}\right), u_{2}$ is defined by this condition.

This section (we shall call it the before-section) is qualitatively similar to the left panel of Fig. 9. Indeed the "ring", trace of the stable manifold $(a)$, cut the trace of the unstable manifold $(h)$ at four points (see Fig. 6). On our section, the unstable manifold $(h)$ is the axis $u_{1}$.

Two "end-loops" $(A)$ and $(B)$ are on the side $u_{3}>0$, and the "area in between" lies on the side $u_{3}<0$. The trajectories starting from the "area in between" will end up in the outside side of the pass; they go to infinity and do not concern us anymore. The area enclosed in the two loops ends up inside the potential well. We can intercept the corresponding trajectories by a new surface of section

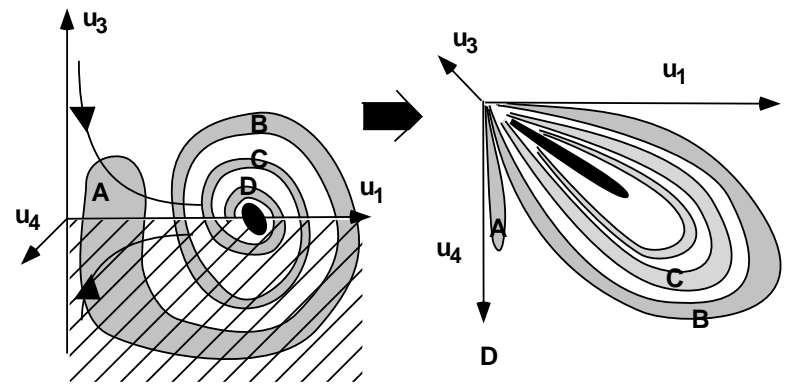

Fig. 10. Passage close to the unstable periodic orbit in the upper right corner, between the second and the third intersections with the surface of section (see Figs. 6 and 7). Comments made concerning Fig. 9 apply here, too

(that we shall call the after-section) defined by $u_{3}=u_{3}^{*}$, where $u_{3}^{*}$ is a given small quantity. The four points of intersection of the ring with the axis $u_{1}$ on the beforesection are sent to the origin and do not appear on the after-section. Hence the two loops $(A)$ and $(B)$ on the after-section are open segments, the boundaries of which is the origin $\left(u_{1}=u_{4}=0\right)$.

To each point on the loops $(A)$ and $(B)$ on the left panel of Fig. 9 a time $\tau$ is associated, with $a \tau=$ $\ln \left(u_{1}(0) / u_{3}^{*}\right)$, the time of flight of the orbit between the two sections. This time goes to infinity when the orbit approaches the heteroclinic orbit (when $u_{1}(0)=0$ ). When we go back to the $x$ coordinates by the inverse of (10), this time $\tau$ will be responsible for the infinite winding around the stable manifold $(d)$.

Indeed, for each $\theta$ (with $0 \leq \theta \leq T$, where $T$ is the period of the unstable periodic orbit), there exists a infinite discrete set of points, converging to $\left(u_{1}=u_{4}=0\right)$, on each end of the curves $(A)$ and $(B)$ such that $(\tau=\theta$, $\bmod T)$. When expressed in the $x$-coordinate system, this set of points converges toward the point

$x=x^{*}(\theta)+\mathcal{T}\left(\theta, 0,0, u_{3}^{*}, 0\right)$

on the unstable manifold. Hence when mapped on the original surface of section $(x=0)$, the sequence of points converges toward the intersection of a particular asymptotic orbit. This is true for any $\theta$ in the interval $[0, T[$, and by continuity, the second section must look as if the loops $(A)$ and $(B)$ of the right panel of Fig. 9 were wound infinitely around the trace of the stable manifold $(d)$ (corresponding to $\left.u_{1}=u_{4}=0\right)$.

Let us consider one of those loops and describe its evolution between the second intersection with the main surface of section and the third (remember that we are going backward in time). From the second intersection we follow it up to the vicinity of the first quadrant guardian (see the left panel of Fig. 10). As it was winding infinitely around the trace of the stable manifold $(d)$, it cut an infinite number of times the trace of the unstable manifold $(e)$ (see Fig. 7). The pieces below the $u_{1}$ axis escape through the upper-right window.

The piece labeled $(A)$ behaves similarly. When mapped on the "after-section" $\left(u_{3}=u_{3}^{*}\right)$, it consists of a loop, the 
two ends of which converge toward the origin $\left(u_{1}=u_{4}=\right.$ $0)$. The same phenomenon occurs when this is translated in the $x$-coordinate system. The loops winds infinitely around the stable manifold $(a)$. This explain the two (they are two loops to start with in Sect. 2) very narrow tongues seen in Fig. 8.

The other pieces in the left panel of Fig. 10 enclose each other. This explain the appearance on the "after-section" of loops embedded in each other. Add to this the usual winding due to the conversion to the $x$-coordinates and we obtain Fig. 8.

The story between the third and fourth intersections could be built from the same building blocks; infinite cuts of the "russian dolls" by the unstable manifold $(h)$, infinite winding around the first intersection of stable manifold (a) (see left panel of Fig. 6), to form a double russian doll system: each doll being itself composed of an infinity of layers. As mentioned above we do not plan to draw such a picture.

\section{Evolution of the spirals}

When the energy approaches the critical energy, the sections of the tubes of escape and injection become smaller and smaller. One would expect then that there will be a cascade of levels of energy at which the stable and unstable manifolds become tangent and the corresponding heteroclinic orbits disappear. The spiral born from this pair of heteroclinic orbits (and all the "russian doll" spirals which have descended from it) becomes finite and the degree of embedding of the russian doll spirals becomes finite.

This is not the case in the problem at hand because of the degeneracy mentioned in Sect. 2. Indeed, if the tubes of escape and injection become smaller, their center become closer and closer. At the limit, the section of the escape tube (resp. the injection tube) is a disk centered on the trace of the one-dimensional stable (resp. unstable) manifold to the equilibrium; but, as we have seen, these two one-dimensional manifolds are the same. Hence, in the problem at hand, the length of the spirals and their degree of embedding remain infinite up to the critical value of the energy

\section{Conclusions}

We have analyzed the shapes and sizes of the windows of escape of stars from a simplified galactic model. We have found that they consist of a "main window" and of a hierarchy of secondary windows. A very large part of the main window is actually made of "just passing through" stars and may not be very interesting for galactic studies.

Indeed the important point for galactic studies is the rate of escape of existing stars. Hence the importance of the secondary windows, their intricate spiral structures and the fractality of the basin boundaries. They govern the rate of escape. A preliminary analysis of this rate of escape, by brute force numerical integration, is to be found in Siopsis et al. (1997). We hope in the future to refine this

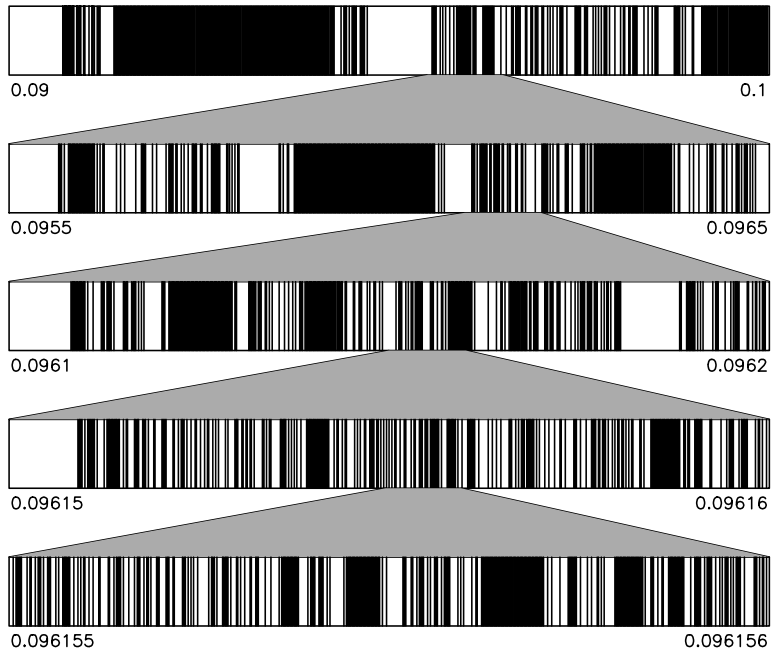

Fig. 11. Direction of escape of orbits starting on the segment $y \in[0.09,0.1], \dot{y}=0.21$. In each box there is a vertical black line if the star escapes through the third quadrant window and a blank line if it escape through the first quadrant one. At each level of zoom the picture is roughly the same

analysis, especially close to the critical value of the energy where the brute force numerical integration is less reliable.

In order to better understand the spiral structures, we have shown how to compute the stable and unstable invariant manifolds of the "guardian" periodic orbits and how to construct a normalized approximation of the flow of a two-degree of freedom Hamiltonian system in the vicinity of an unstable periodic orbit.

Previous numerical or analytical investigations have already shown that homoclinic or heteroclinic orbits generate spirals in Poincaré sections, but our analysis, based on the above-mentioned normalization, describes in more detail the complex layered nature of these spirals. There are "first-order", infinitely winding spirals as described in (Contopoulos \& Kaufmann 1992), but also "second order" spirals composed themselves of an infinity of layers, "third order" spirals formed by an infinity of second order spirals, and so on.

The existence of those multi-order spirals is, of course, associated with the fractal nature of the boundary between classes of motion. Chaotic scattering problems repeatedly show this behavior (see for instance Ott 1993). Figure 11 is an illustration of this fractality. We have indicated the window of escape of orbits corresponding to regularly spaced initial conditions on a segment in the plane $(y, \dot{y})$ for $h=0.05$. Zooming five times by a power of ten on this segment, we see the pattern repeating itself, implying a "self similarity" of the boundary between the two classes of motion.

\section{Appendix 1}

Let us consider a Hamiltonian linear system, the coefficients of which are periodic of period $T$ in time

$\dot{\boldsymbol{y}}=\Im A(t) \boldsymbol{y}$, 
where $A(t)$ is a symmetric periodic matrix. Let us also consider its principal matrix $\Psi(t)$,

$\dot{\Psi}=\Im A(t) \Psi, \quad$ with $\quad \Psi(0)=I d$.

The principal matrix $\Psi(t)$ is symplectic, as is the monodromy matrix $\Psi(T)$. It is easy to show that the matrix

$R=\frac{1}{T} \ln \Psi^{-1}(T)$,

is Hamiltonian (i.e. such that $\Im R=R^{T} \Im$ ). Indeed the exponential of any Hamiltonian matrix is symplectic and vice-versa.

Hence the reduction of the periodic linear system to a constant linear system

$\dot{z}=\Im B z$,

by means of the linear transformation $\boldsymbol{y}=Q(t) \boldsymbol{z}$, with

$Q(t)=\Psi(t) \exp R t$

is symplectic.

It remains to be seen that $B$ can take the particular form shown in (13). We know that zero is a double eigenvalue with only one eigenvector (because the period of the periodic orbit is not stationary along the family of periodic orbits - see Deprit \& Henrard 1968), and that the other two eigenvalues are $a$ and $-a$. We can then apply the construction proposed by Laub \& Meyer (1974) for building a normal form for Hamiltonian linear systems.

\section{Appendix II}

Let us assume that the system derived from the Hamiltonian

$\mathcal{H}\left(x_{1}, x_{2}, x_{3}, x_{4}\right)$,

where $\left(x_{3}, x_{4}\right)$ are the momenta conjugate to $\left(x_{1}, x_{2}\right)$, possesses a family of unstable periodic orbits parameterized by the value $(h)$ of the Hamiltonian. We assume that, for a particular value $h^{*}$ of $h$, the period $T^{*}$ of the orbit $\left(x_{i}^{*}\right)$ is not stationary (i.e. $\left.(\mathrm{d} T / \mathrm{d} h)^{*} \neq 0\right)$, which is indeed the case for the periodic orbit we are investigating.

By Floquet theory we know that the variational equation

$\dot{\boldsymbol{y}}=\Im \mathcal{H}_{x x}^{*}(t) \boldsymbol{y}$,

can be reduced through a periodic linear transformation $\boldsymbol{y}=Q(t) \boldsymbol{z}$ to a linear system with constant coefficients. We recall in the appendix that this can be achieved by means of a symplectic matrix $Q(t)$, leading to a Hamiltonian linear system with constant coefficients

$\dot{z}=\Im \mathcal{N} z$

Furthermore, we can assume (see Appendix I) that the matrix $\Im \mathcal{N}$ is under a special normal form, the equivalent, for Hamiltonian matrix to the Jordan normal form. We know that $\Im \mathcal{N}$ has two real eigenvalues $(a,-a)$ and one, and only one (because $\mathrm{d} T / \mathrm{d} h \neq 0$ ) eigenvector corresponding to a double zero-eigenvalue (see for instance Deprit \& Henrard 1967). Hence the normal form of $\mathcal{N}$ is

$\mathcal{N}=\left(\begin{array}{cccc}0 & 0 & a & 0 \\ 0 & -1 & 0 & 0 \\ a & 0 & 0 & 0 \\ 0 & 0 & 0 & 0\end{array}\right)$

Expanding the Hamiltonian function (11) in powers of the components of $\boldsymbol{z}$, we obtain the "new Hamiltonian":

$\mathcal{K}=a z_{1} z_{3}-\frac{1}{2} z_{2}^{2}+\sum_{i \geq 1} K_{i}\left(t, z_{1}, z_{2}, z_{3}, z_{4}\right)$

where $K_{i}$ is a polynomial of degree $(i+2)$ in the components of $\boldsymbol{z}$ and a periodic function of period $T^{*}$ in $t$.

We shall define a formal canonical transformation from $\boldsymbol{z}$ to $\boldsymbol{u}$ which "normalizes" the Hamiltonian (15). This normalizing transformation will be performed in two steps, each step being defined by a canonical Lie transformation. We assume that the reader is familiar enough with the Lie transformation (Deprit 1969) method to recall that it hinges upon the solution of the homological equation (see for instance Arnold 1988)

$-\left(K_{0} ; \mathcal{W}_{k}\right)+\frac{\partial}{\partial t} \mathcal{W}_{k}=\tilde{K}_{k}-K^{k}$

where $K_{0}$ is the part of degree 2 in $(15), \mathcal{W}_{k}$ is the order $k$ of the generating function, $\tilde{K}_{k}$ is at each order a known function, periodic of period $T^{*}$ in $t$ and polynomial of degree $(k+2)$ in the components of $\boldsymbol{z}$. In order to "solve" (16) we have to choose $K^{k}$ in such a way that the right hand member of (16) belongs to the image of the linear operator

$$
\begin{aligned}
\mathcal{L} \mathcal{W}_{k} & =-\left(K_{0} ; \mathcal{W}_{k}\right)+\frac{\partial}{\partial t} \mathcal{W}_{k} \\
& =\left\{a z_{1} \frac{\partial}{\partial z_{1}}-a z_{3} \frac{\partial}{\partial z_{3}}-2 z_{2} \frac{\partial}{\partial z_{2}}+\frac{\partial}{\partial t}\right\} \mathcal{W}_{k}
\end{aligned}
$$

acting on functions $\mathcal{W}_{k}$, periodic of period $T^{*}$ in $t$ and polynomial of degree $(k+2)$ in the components of $\boldsymbol{z}$.

In the first step we shall show that we can always choose $K^{k}$ in the subspace of functions of the form $F\left(Z, z_{2}, z_{4}\right)$ where $Z=z_{1} z_{3}$.

Indeed let us first show that for any function of the form:

$G_{k}^{i}=z_{4}^{i} \sum_{p, q, r} \sum_{j} z_{1}^{p} z_{2}^{q} z_{3}^{r}\left[C_{p q r j}^{k i} \cos j \omega t+D_{p q r j}^{k i} \sin j \omega t\right]$

we can solve the auxiliary homological equation:

$\mathcal{L}_{1} W_{k}^{i}=\left\{a z_{1} \frac{\partial}{\partial z_{1}}-a z_{3} \frac{\partial}{\partial z_{3}}+\frac{\partial}{\partial t}\right\} W_{k}^{i}=G_{k}^{i}-K^{k}$,

with $K^{k}$ of the form mentioned above.

Take for $W_{k}^{i}$ a function of the same form as $G_{k}^{i}$ :

$W_{k}^{i}=z_{4}^{i} \sum_{p, q, r} \sum_{j} z_{1}^{p} z_{2}^{q} z_{3}^{r}\left[A_{p q r j}^{k i} \cos j \omega t+B_{p q r j}^{k i} \sin j \omega t\right]$ 
the auxiliary homological Eq. (19) leads to a set of linear systems of the form:

$$
\left\{\begin{array}{c}
(p-r) A_{p q r j}^{k i}+j \omega B_{p q r j}^{k i}=C_{p q r j}^{k i} \\
-j \omega A_{p q r j}^{k i}+(p-r) B_{p q r j}^{k i}=D_{p q r j}^{k i} .
\end{array}\right.
$$

These linear systems can easily be solved, provided the determinants $(p-r)^{2}+j^{2} \omega^{2}$ do not vanish. The determinants vanish only when both $(p-r)$ and $j$ are zero. Hence the form we propose for $K^{k}$.

Now let us expand $\tilde{K}_{k}$ in Fourier series

$\tilde{K}_{k}=\sum_{i=0}^{k+2} K_{k}^{i}$

with

$\tilde{K}_{k}^{i}=z_{4}^{i} \sum_{p, q, r} \sum_{j} z_{1}^{p} z_{2}^{q} z_{3}^{r}\left[E_{p q r j}^{k i} \cos j \omega t+F_{p q r j}^{k i} \sin j \omega t\right]$,

Starting with $i=k+2$, we define $W_{k}^{k+2}$ as the solution of $\mathcal{L}_{1} W_{k}^{k+2}=\tilde{K}_{k}^{k+2}-K_{k}^{k+2}$. We have:

$$
\begin{aligned}
\mathcal{L} W_{k}^{k+2} & =\mathcal{L}_{1} W_{k}^{k+2}-2(k+2) z_{2} z_{4}^{-1} W_{k}^{k+2} \\
& =\tilde{K}_{k}^{k+2}-K_{k}^{k+2}-2(k+2) z_{2} z_{4}^{-1} W_{k}^{k+2} .
\end{aligned}
$$

Hence the homological Eq. (16) is solved as far as terms of degree $k+2$ in $z_{4}$ are concerned; of course we have generated extra terms of degree $k+1$ in $z_{4}$. They will be removed at the same time that $\tilde{K}_{k}^{k+1}$ is normalized. Therefore, we take

$G_{k}^{k+1}=\tilde{K}_{k}^{k+1}+2(k+2) z_{2} z_{4}^{-1} W_{k}^{k+2}$,

and define $W_{k}^{k+1}$ as the solution of the auxiliary homological equation $\mathcal{L}_{1} W_{k}^{k+1}=G_{k}^{k+1}-K_{k}^{k+1}$. In this way the homological Eq. (16) is solved as far as terms of degree $k+2$ and $k+1$ in $z_{4}$ are concerned, and we have generated extra terms of degree $k$ in $z_{4}$. They in turn can be removed while examining the terms of degree $k$ and so on. The recurrence ends when we solve for the terms of degree zero in $z_{4}$. For those terms $\mathcal{L}=\mathcal{L}_{1}$ and no extra terms are generated.

After the transformation from the phase space $\left(z_{1}, z_{2}, z_{3}, z_{4}\right)$ to the phase space $\left(w_{1}, w_{2}, w_{3}, w_{4}\right)$ generated by the generating function $\mathcal{W}=\sum \mathcal{W}_{k}$ the Hamiltonian function is reduced to

$\mathcal{M}=a W-\frac{1}{2} w_{2}^{2}+\sum_{i \geq 1} M_{i}\left(W, w_{2}, w_{4}\right)$,

where $W$ stands for the product $\left(w_{1} w_{3}\right)$. A second transformation, from the phase space $\left(w_{1}, w_{2}, w_{3}, w_{4}\right)$ to the phase space $\left(u_{1}, u_{2}, u_{3}, u_{4}\right)$, will eliminate the dependency on $u_{2}$. Due to the fact that the function space we are interested in, no longer depends on the time and depends on the coordinates $\left(w_{1}, w_{3}\right)$ only through their product $W$, the homological equations reduces to

$-\left(\mathcal{M}_{0} ; \mathcal{V}_{k}\right)=-2 w_{2} \frac{\partial}{\partial w_{4}} V_{k}=\tilde{M}_{k}-M^{k}$

If we expand

$\tilde{M}_{k}=\sum_{p q r} A_{p q r} W^{p} w_{2}^{q} w_{4}^{r}$,

the solution is simply given by

$M^{k}=\sum_{p r} A_{p 0 r} W^{p} w_{4}^{r}$,

$\mathcal{V}_{k}=\sum_{p q r}^{r \neq 0} \frac{-1}{2(r+1)} A_{p q r} W^{p} w_{2}^{q-1} w_{4}^{r+1}$.

Eventually the normalized Hamiltonian reads

$\mathcal{P}=a U-\mathcal{R}\left(U, u_{2}, u_{4}\right), \quad \mathcal{R}=\frac{1}{2} u_{2}^{2}+\sum_{i \geq 1} R_{i}\left(U, u_{4}\right)$,

the product $U=u_{1} u_{3}$ and the function $\mathcal{R}$ are both constants of the motion, equal to zero on the stable and unstable manifolds. The function $\mathcal{R}$ can be considered as the Hamiltonian of a one degree of freedom problem depending on the parameter $U$. Notwithstanding the fact that the algorithms proposed do not generate small denominators, we do not know whether the generating formal power series $\mathcal{W}=\sum \mathcal{W}_{k}$ and $\mathcal{V}=\sum \mathcal{V}_{k}$ converge. But as we will use the results of this normalization only for developing good approximations of the motion in the vicinity of the unstable periodic orbit, a truncated normalization will suffice.

\section{References}

Arnold, V. I. 1988, Dynamical Systems III (Springer-Verlag) Barbanis, B. 1990, Celest. Mech Dyn. Astr., 48, 57

Bleher, S., Grebogi, C., \& Ott, E. 1990, Physica D, 46, 87

Contopoulos, G. 1990, A\&A, 231, 41

Contopoulos, G., \& Kaufmann, D. 1992, A\&A, 253, 379

Deprit, A. 1969, Celest. Mech. Dyn. Astr., 1, 308

Deprit, A., \& Henrard, J. 1968, Adv. Astron. Astrophys., 6, 1 Deprit, A., \& Henrard, J. 1969, AJ, 74, 308

Deprit, A., \& Price, J. F. 1969, A\&A, 1, 427

Hénon, M., \& Heiles, C. 1964, AJ, 69, 73

Henrard, J., \& Navarro, J. F. 2001, Celest. Mech. Dyn. Astr., accepted for publication

Laub, A. J., \& Meyer, K. 1974, Celest. Mech. Dyn. Astr., 9, 213

Moser, J. 1973, Stable and Random Motions in Dynamical Systems with Special Emphasis on Celestial Mechanics, Princeton U.P.

Noid, D. W., Gray, S. K., \& Stuart, A. R. 1985, J. Chem. Phys., 84,2649

Petit, J. M., \& Hénon, M. 1986, Icarus, 66, 536

Poincaré, H. 1899, Méthodes nouvelles de la mécanique céleste, Tome III (Gauthier-Villars)

Ott, E. 1993, Chaos in Dynamical Systems (Cambridge U.P.)

Siopsis, C., Kandrup, H. E., Contopoulos, G., \& Dvorak, R. 1997, Celest. Mech. Dyn. Astr., 65, 57

Verhulst, F. 1979, Phil. Trans. Royal Soc. London, 290, 435 